\title{
Gender Differences in Somatic Symptoms and Current Suicidal Risk in Outpatients with Major Depressive Disorder
}

\author{
Hong Jin Jeon ${ }^{1,2}$, Jong-Min Woo ${ }^{3,4}$, Hyo-Jin Kim5, Maurizio Fava², David Mischoulon², Seong Jin Cho ${ }^{6}$, \\ Sung Man Chang ${ }^{7}$, Doo-Heum Park ${ }^{8}$, Jong Woo Kim ${ }^{9}$, Ikki Yoo', Jung-Yoon Heo', and Jin Pyo Hong ${ }^{1 凶}$ \\ ${ }^{1}$ Department of Psychiatry, Depression Center, Samsung Medical Center, Sungkyunkwan University School of Medicine, Seoul, Republic of Korea \\ 2Depression Clinical and Research Program, Massachusetts General Hospital, Harvard Medical School, Boston, MA, USA \\ ${ }^{3}$ Department of Psychiatry, Seoul Paik Hospital, Inje University School of Medicine, Seoul, Republic of Korea \\ ${ }^{4}$ Stress Research Institute, Inje University, Seoul, Republic of Korea \\ ${ }^{5}$ OR/RWD Team, Corporate Affairs·Health \& Value Division, Pfizer Korea, Seoul, Republic of Korea \\ ${ }^{6}$ Department of Psychiatry, Gil Medical Center, Gachon Medical School, Incheon, Republic of Korea \\ ${ }^{7}$ Department of Psychiatry, Kyungpook National University Hospital, Kyungpook National University School of Medicine, Daegu, Republic of Korea \\ ${ }^{8}$ Department of Psychiatry, Konkuk University School of Medicine, Seoul, Republic of Korea \\ ${ }^{9}$ Department of Psychiatry, Kyung Hee University Hospital, College of Medicine, Kyung Hee University, Seoul, Republic of Korea
}

Objective Although somatic symptoms are common complaints of patients with major depressive disorder (MDD), their associations with suicide are still unclear.

Methods A total of 811 MDD outpatients of aged between 18 to 64 years were enrolled nationwide in Korea with the suicidality module of the Mini-International Neuropsychiatric Interview (MINI) and the Depression and Somatic Symptom Scale (DSSS).

Results On stepwise regression analysis, current suicidality scores were most strongly associated with chest pain in men, and neck or shoulder pain in women. Severe chest pain was associated with higher current suicidality scores in men than in women, whereas severe neck or shoulder pain showed no significant differences between the genders. In conclusion, MDD patients of both sexes with suicidal ideation showed significantly more frequent and severe somatic symptoms than those without. Current suicidal risk was associated with chest pain in men, and neck or shoulder pain in women.

Conclusion We suggest that clinicians pay attention to patients' somatic symptoms in real world practice.

Psychiatry Investig 2016;13(6):609-615

Key Words Somatic symptom, Suicidality, Gender difference, Chest pain.

\section{INTRODUCTION}

Somatic symptoms are prevalent in patients with major depressive disorder (MDD). Symptoms include headache, back pain, neck and shoulder pain, and muscle tension, especially in the primary care setting. ${ }^{1}$ Although they are not included as diagnostic criteria in the Diagnostic and Statistical Manual of Mental Disorders (DSM-5), ${ }^{2}$ somatic symptoms sometimes

Received: April 19, 2015 Revised: November 10, 2015

Accepted: November 22, 2015 Available online: November 2, 2016

$\triangle$ Correspondence: Jin Pyo Hong, MD, PhD

Department of Psychiatry, Sungkynkwan University School of Medicine, Samsung Medical Center, 81 Irwon-ro Gangnam-gu, Seoul 06351, Republic of Korea Tel: +82-2-3410-3585, Fax: +82-2-3410-0050, E-mail: suhurhong@gmail.com

@ This is an Open Access article distributed under the terms of the Creative Commons Attribution Non-Commercial License (http://creativecommons.org/licenses/bync/3.0) which permits unrestricted non-commercial use, distribution, and reproduction in any medium, provided the original work is properly cited. present as the chief complaint of depression in primary care settings. ${ }^{3}$ Previous studies revealed gender differences in somatic symptoms of MDD patients. Among MDD patients, women have double the rates of somatic symptoms as men, ${ }^{4}$ and the prevalence of somatic depression as opposed to depression without somatic symptoms, was much higher among women than men. ${ }^{5}$

MDD is a major risk factor for suicide. ${ }^{6}$ However, not all individuals with major depression commit suicide. Many studies have investigated factors associated with suicide in MDD patients, such as psychiatric comorbidities, ${ }^{6,7}$ genetic factors, ${ }^{8,9}$ melancholic features of MDD, ${ }^{10,11}$ biomarkers, ${ }^{12}$ and environmental factors. ${ }^{13}$ Although some studies suggested that somatic symptoms are related to suicide, ${ }^{14,15}$ gender differences regarding somatic symptoms and suicide are still poorly understood in MDD patients. 
We hypothesized that MDD patients with more severe somatic symptoms may have more suicidal ideation, and gender differences may be found in the types of somatic symptoms related to current suicidal risk. We investigated which somatic symptoms were most strongly associated with suicidality score in MDD patients of each gender, and the number of somatic symptoms and their relationship to suicidal risk.

\section{METHODS}

\section{Data source, data collection, and study sample}

Data from Korean MDD patients were derived from the Burden of illness in Major depressive disorder Study. This was a cross-sectional, multicenter-based, naturalistic, outpatientbased observational study of MDD. They were enrolled from 14 regional centers which were psychiatric outpatient clinics throughout Korea. The study was conducted from December 2011 to September 2012. All study procedures were approved by the Institutional Review Board of the Samsung Medical Center and each site. Institutional review board-approved written informed consent was obtained from all study patients before commencing the study.

The regional centers were as follows: three sites in the South districts of Seoul; 1) Samsung Medical Center, Sungkyunkwan University School of Medicine, 2) Asan Medical Center, University of Ulsan College of Medicine, 3) Seoul St. Mary's Hospital, The Catholic University College of Medicine, three sites in the north district of Seoul, 4) Seoul Paik Hospital, Inje University, 5) Konkuk University Medical Center, 6) Kyung Hee University Medical Center, two sites in Incheon, 7) Ajou University Hospital, 8) Gil Medical Center, Gachon University, two sites in Daejeon, 9) Chungnam National University Hospital, 10) Eulji University Hospital, one site in Cheonan, 11) Dankook University Hospital, one site in Daegu, 12) Kyungpook National University Hospital, 13) one site in Busan, Dong-A University Medical Center, and one site in Gyeongju, and 14) Dongguk University Gyeongju Hospital.

Inclusion criteria were aged between 18 to 64 years, and fulfillment of the DSM-IV criteria for single or recurrent nonpsychotic MDD. ${ }^{16}$ The 17 -item Hamilton Depression Rating Scales (HAM-D) were applied to the patients. ${ }^{17}$ Exclusion criteria were a history of bipolar disorder, schizophrenia, schizoaffective disorder, psychosis, anorexia or bulimia nervosa, obsessive compulsive disorder, and serious general medical conditions. Participants met DSM-IV criteria for nonpsychotic major depressive disorder at study entry, as determined by the clinical diagnosis and confirmed with a DSM-IV diagnosis by a psychiatrist or clinical research coordinator.

\section{Measures}

The Depression and Somatic Symptoms Scale (DSSS) was applied to measure the somatic symptoms of the MDD patients. ${ }^{18}$ It is a 22 -item self-administered rating scale, including three subscales as the Depression Subscale (DS), Pain Subscale (PS), and Somatic Subscale (SS). The DS had 12 items, including three vegetative symptoms and fatigue, and the SS had 10 items, including five pain items, which comprised the 5 -item pain subscale (PS). Each item was rated with a 0-3 score: 0 (not at all), 1 (mild), 2 (moderate), and 3 (severe). The range of the sum score was thus $0-66$. The scale had good validity and reliability, and the higher the score the more severe the symptoms. ${ }^{19}$ The Korean Version of the DSSS showed high reliability $(r=0.83, \mathrm{p}<0.01)$ and validity (Cronbach's alpha 0.90). ${ }^{20}$

Current suicidal risk was assessed with the Korean version of the MINI suicidality module. ${ }^{21}$ The MINI suicidality module was composed of six questions with a different weight: wish for death (1), wish for self-harm (2), suicidal thought (6), suicide plan (10), suicide attempt in the past month (10), and lifetime suicide attempt (4). Recent suicide-related questions were based on symptoms during the past 1 month. The MINI suicidality score was estimated from the sum of the weighted score of the six questions.

\section{Statistical analysis}

We performed t-tests and chi-square tests for demographic and clinical profiles of the MDD patients between the genders. The somatic symptoms were compared between those with and without suicidal ideation in each gender with Analysis of Covariance (ANCOVA) that was used to control for the HAM$\mathrm{D}$ scores in order to adjust for the severity of depression between the two groups. The association between severity of each somatic symptom and current suicidality scores was evaluated by backward multiple linear regression using the full set of variables of the Depression and Somatic Symptom Scale, total HAM-D score and age. Then, we selected the somatic symptoms which were strongly associated with current suicidality scores for each gender, and plotted them as a multiple line graph according to the scores of subjective somatic symptoms. Current suicidality scores were compared between the genders for each score of the somatic symptoms and for each number of somatic symptoms with Student's t-test. All analyses were two-tailed, and calculations were performed using SAS $^{\circledR}$ Version 9.2, SAS institute, Cary, NC, USA.

\section{RESULTS}

\section{Demographics and clinical profiles}

Male MDD patients showed significantly younger age, were 
Table 1. Demographics and clinical profiles $(\mathrm{N}=811)$

\begin{tabular}{|c|c|c|c|c|}
\hline \multirow{2}{*}{ Variables } & \multicolumn{3}{|c|}{ MDD patients } & \multirow{2}{*}{ Statistics $\mathrm{p}$} \\
\hline & Total $(\mathrm{N}=811)$ & Men $(\mathrm{N}=231)$ & Women $(\mathrm{N}=580)$ & \\
\hline Age (years) & $45.5 \pm 13.03$ & $41.9 \pm 14.18$ & $46.9 \pm 12.26$ & $<0.001^{\mathrm{a}}$ \\
\hline \multicolumn{5}{|l|}{ Education years (\%) } \\
\hline $0-6$ & $118(14.5)$ & $21(9.1)$ & $97(16.7)$ & \multirow[t]{4}{*}{$<0.001^{\mathrm{b}}$} \\
\hline $7-9$ & $120(14.8)$ & $25(10.8)$ & $95(16.4)$ & \\
\hline $10-12$ & $333(41.1)$ & $92(39.8)$ & $241(41.6)$ & \\
\hline$\geq 13$ & $239(29.5)$ & $93(40.3)$ & $146(25.2)$ & \\
\hline \multicolumn{5}{|l|}{ Marital status, n (\%) } \\
\hline Married & $531(65.5)$ & $119(51.5)$ & $412(71.0)$ & \multirow[t]{3}{*}{$<0.001^{\mathrm{b}}$} \\
\hline Single & $161(19.9)$ & $84(36.4)$ & $77(13.3)$ & \\
\hline Widowed/divorced/separated & $118(14.5)$ & $28(12.1)$ & $90(15.5)$ & \\
\hline \multicolumn{5}{|l|}{ Previous one month (\%) } \\
\hline Suicidal ideation & $262(32.3)$ & $79(34.2)$ & $183(31.6)$ & \multirow[t]{2}{*}{$0.124^{\mathrm{b}}$} \\
\hline Suicidal attempt & $45(5.5)$ & $18(7.8)$ & $27(4.7)$ & \\
\hline Current suicidality scores & $9.5 \pm 8.47$ & $10.8 \pm 9.11$ & $9.0 \pm 8.14$ & $0.033^{\mathrm{a}}$ \\
\hline HAM-D scores & $13.5 \pm 8.02$ & $14.1 \pm 7.97$ & $13.3 \pm 8.03$ & $0.170^{\mathrm{a}}$ \\
\hline Onset of MDD (years) & $42.4 \pm 13.81$ & $39.2 \pm 15.09$ & $43.7 \pm 13.06$ & $<0.001^{\mathrm{a}}$ \\
\hline Number of depressive episodes & $1.4 \pm 2.28$ & $1.2 \pm 2.01$ & $1.4 \pm 2.38$ & $0.163^{\mathrm{a}}$ \\
\hline Duration of depression (months) & $7.2 \pm 17.19$ & $5.8 \pm 12.84$ & $7.8 \pm 18.62$ & $0.090^{\mathrm{a}}$ \\
\hline
\end{tabular}

adifference between genders (two sample t-test), bdifference between genders (chi-square test). MDD: major depressive disorder, HAM-D: Hamilton Depression Rating Scale

Table 2. Comparisons of somatic symptoms in MDD subjects with and without suicidal attempt and ideation within the previous one month $(\mathrm{N}=811)$

\begin{tabular}{|c|c|c|c|c|c|c|c|c|}
\hline \multirow[b]{2}{*}{ Somatic symptoms (DSSS) } & \multicolumn{4}{|c|}{$\operatorname{Men}(\mathrm{N}=231)$} & \multicolumn{4}{|c|}{ Women $(\mathrm{N}=580)$} \\
\hline & $\%$ & $\begin{array}{c}\text { With suicidal } \\
\text { ideation }(\mathrm{N}=97) \text {, } \\
\text { LS Mean }(\mathrm{SE})\end{array}$ & $\%^{\mathrm{a}}$ & 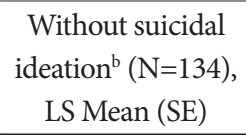 & $\%$ & $\begin{array}{c}\text { With suicidal } \\
\text { ideation }(\mathrm{N}=210) \text {, } \\
\text { LS Mean }(\mathrm{SE})\end{array}$ & $\%^{\mathrm{a}}$ & 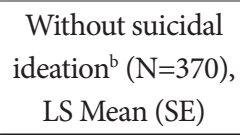 \\
\hline Headache & 78.4 & $1.04(0.09)$ & $47.8^{* * *}$ & $0.78(0.08)^{*}$ & 76.2 & $1.05(0.06)$ & $55.7^{* * *}$ & $0.92(0.05)$ \\
\hline Chest tightness & 86.6 & $1.33(0.08)$ & $53.0^{* * *}$ & $0.95(0.07)^{* * *}$ & 87.6 & $1.31(0.06)$ & $57.0^{* * *}$ & $1.05(0.05)^{* * *}$ \\
\hline Muscle tension & 74.2 & $1.15(0.09)$ & $47.8^{* * *}$ & $0.84(0.08)^{*}$ & 74.3 & $1.10(0.06)$ & $51.6^{* * *}$ & $0.86(0.05)^{* *}$ \\
\hline Back pain & 56.7 & $0.58(0.08)$ & $38.1^{* *}$ & $0.71(0.07)$ & 64.8 & $0.90(0.06)$ & $50.5^{* *}$ & $0.81(0.05)$ \\
\hline Dizziness & 74.2 & $1.03(0.09)$ & $47.8^{* * *}$ & $0.74(0.07)^{*}$ & 73.8 & $0.99(0.06)$ & $57.0^{* * *}$ & $0.83(0.04)^{*}$ \\
\hline Chest pain & 62.9 & $0.81(0.08)$ & $33.6^{* * *}$ & $0.53(0.07)^{*}$ & 62.9 & $0.72(0.06)$ & $40.0^{* * *}$ & $0.66(0.04)$ \\
\hline Neck or shoulder pain & 73.2 & $1.05(0.10)$ & $54.5^{* *}$ & $1.01(0.08)$ & 80.0 & $1.24(0.07)$ & $66.8^{* *}$ & $1.13(0.05)$ \\
\hline Shortness of breath & 57.7 & $0.74(0.08)$ & $32.8^{* *}$ & $0.50(0.06)^{*}$ & 62.4 & $0.73(0.05)$ & $33.0^{* * *}$ & $0.53(0.04)^{* *}$ \\
\hline $\begin{array}{l}\text { Soreness in more than half } \\
\text { of the body's muscles }\end{array}$ & 55.7 & $0.67(0.08)$ & $28.4^{* * *}$ & $0.47(0.06)$ & 62.4 & $0.79(0.06)$ & $45.9^{* * *}$ & $0.80(0.04)$ \\
\hline Palpitation & 83.5 & $1.13(0.08)$ & $46.3^{* * *}$ & $0.77(0.07)^{* *}$ & 78.1 & $1.08(0.06)$ & $53.2^{* * *}$ & $0.86(0.04)^{* *}$ \\
\hline Total DSSS scores & & $27.29(0.93)$ & & $19.50(0.77)^{* * *}$ & & $25.73(0.65)$ & & $20.71(0.48)^{* * *}$ \\
\hline
\end{tabular}

${ }^{a}$ percent of patients who experienced each somatic symptom, and chi-square test results between those with and without suicidal ideation for each gender (two-tailed), badjusted for total HAM-D scores (ANCOVA analyses). ${ }^{*} \mathrm{p}<0.05,{ }^{* *} \mathrm{p}<0.01,{ }^{* * *} \mathrm{p}<0.001$. MDD: major depressive disorder, DSSS: The Depression and Somatic Symptoms Scale, LS mean: least-squares mean, ANCOVA: analysis of covariance 
more highly educated, more often single, and had an earlier onset of MDD than female MDD patients (Table 1). There were no statistically significant differences between genders in suicidal ideation and attempts in the previous month, current suicidality scores, HAM-D scores, number of depressive episodes, and duration of depression.

\section{Somatic symptoms and suicidal ideation}

MDD patients, in both men and women, with suicidal ideation showed significantly higher proportion of all somatic symptoms of DSSS questionnaire by chi-square test and higher total DSSS scores by ANCOVA with adjustment for total HAM-D scores than those without suicidal ideation (Table 2). For the frequency of each item of the DSSS, MDD patients with suicidal ideation showed a significantly higher frequency of somatic symptoms than those without suicidal ideation for all somatic symptoms in each gender. For the mean values of each item, MDD patients with suicidal ideation showed signif- icantly higher somatic symptom scores than those without suicidal ideation, with symptoms such as chest tightness, muscle tension, dizziness, shortness of breath, and palpitations reported in both genders. No significant differences were found in back pain, neck or shoulder pain, and soreness in more than half of the body's muscles between those with and without suicidal ideation in both genders.

\section{Somatic symptoms and current suicidal risk}

Table 3 presents backward multiple linear regression analysis of somatic symptoms for current suicidality score in each gender. Current suicidality scores were significantly associated with chest pain $(\beta=2.40, p=0.004)$ and muscle tension $(\beta=1.65$, $\mathrm{p}=0.020$ ) in men, whereas suicidality scores were associated with neck or shoulder pain $(\beta=1.08, \mathrm{p}=0.016)$ and chest tightness $(\beta=0.92, p=0.043)$ in women. On the other hand, current suicidality scores showed no significant association with both chest pain and muscle tension in women, or with both neck or

Table 3. Results of stepwise backward multiple linear regression ${ }^{\mathrm{a}}$ analysis: association between somatic symptoms and current suicidality scores in MDD patients by gender

\begin{tabular}{|c|c|c|c|c|}
\hline \multirow{3}{*}{ Variables } & \multicolumn{4}{|c|}{ Current suicidality scores } \\
\hline & \multicolumn{2}{|c|}{$\operatorname{Men}(\mathrm{N}=231)$} & \multicolumn{2}{|c|}{ Women $(\mathrm{N}=580)$} \\
\hline & Coefficient $(\beta)$ & $\mathrm{p}$ & Coefficient $(\beta)$ & $\mathrm{p}$ \\
\hline Chest pain & 2.40 & 0.004 & - & - \\
\hline Muscle tension & 1.65 & 0.020 & - & - \\
\hline Neck or shoulder pain & - & - & 1.08 & 0.016 \\
\hline Chest tightness & - & - & 0.92 & 0.043 \\
\hline
\end{tabular}

adjusted for age. MDD: major depressive disorder



Figure 1. Severity of each somatic symptom and current suicidality scores in MDD Patients ( $\mathrm{N}=811)$. Student's t-test of current suicidality scores between the genders in each score of the somatic symptoms among the patients who had chest pain. ${ }^{*} p<0.05$. MDD: major depressive disorder.

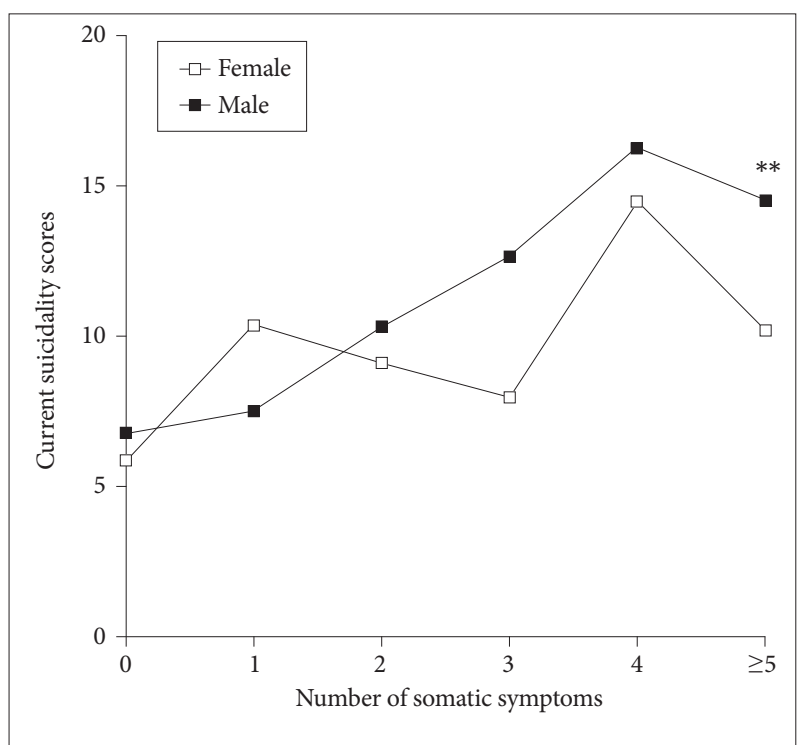

Figure 2. The number of somatic symptoms and current suicidality scores by gender $(\mathrm{N}=811)$. Student's t-test of current suicidality scores between the genders for each number of somatic symptoms. ${ }^{* *} p<0.01$. 
shoulder pain and chest tightness in men.

\section{Severity of somatic symptoms and current suicidal risk}

Severe chest pain was associated with higher current suicidality scores in men than women in the scores of $2(\mathrm{t}=2.53$, $\mathrm{p}=0.014)$ and $3(\mathrm{t}=2.65, \mathrm{p}=0.013)$ for chest pain, but no significant differences in scores of 0 and 1 were observed (Figure 1). In contrast, severe neck or shoulder pain showed no difference between the genders for all somatic symptom scores. Men showed significantly higher current suicidality scores than women in those with more than five symptoms $(\mathrm{t}=2.72, \mathrm{p}=$ 0.007) (Figure 2).

\section{DISCUSSION}

This is the first study to investigate the association between somatic symptoms and suicidal risk in Korean MDD patients by gender. We report two main findings. First, both male and female MDD patients with suicidal ideation showed significantly more frequent and severe somatic symptoms than those without suicidal ideation. Second, somatic symptoms are strongly associated with current suicidal risk in MDD patients, and there were gender differences in the types of somatic symptoms which were most strongly associated with current suicidal risk: chest pain was most strongly associated in men, and neck or shoulder pain in women.

This study adds to the current literature on depression by showing that somatic symptoms were more frequent and severe in patients with MDD with suicidal ideation than those without suicidal ideation in both genders; symptoms reported were headache, chest tightness, muscle tension, back pain, dizziness, chest pain, neck or shoulder pain, shortness of breath, soreness, and palpitation, supporting the previous finding that somatic symptoms were prevalent in MDD patients. ${ }^{1}$ The severity of somatic symptoms showed gender differences in that male MDD patients with suicidal ideation showed a higher incidence of chest pain and headache than those without suicidal ideation, but women did not. Previous studies showed that somatic complaints were highly associated with depression and anxiety ${ }^{22}$ in both men and women. ${ }^{23}$ Somatic symptoms are associated with a higher risk of early relapse, chronicity, suicide, and mortality due to other natural causes, and result in poor prognosis, functional status and health-related quality of life in depressed patients. ${ }^{3}$ From the results of the STAR*D study, patients who experienced somatic symptoms exhibited lower remission rates in response to antidepressant treatment. ${ }^{24}$

These results are consistent with previous studies that showed that pain was an independent risk factor for suicide and high comorbidity with depression, in population-based studies, ${ }^{25,26}$ and clinical studies on patients with cancer, ${ }^{27} \mathrm{mi}$ graine, ${ }^{28}$ and fibromyalgia. ${ }^{29}$ However, few previous studies have focused on the pain of clinical MDD patients. This study revealed that pain is frequently found in clinical MDD patients, and pain is strongly associated with current suicidal risk. Previous studies have demonstrated that more than half of depressed patients report physical complaints only, and at least $60 \%$ of these complaints refer to pain. ${ }^{30}$ Among somatic symptoms, severe and chronic pain is associated with a poor prognosis of depression. Pain is a strong predictor of a longer time to remission and may be a marker of a more difficult-totreat depression, ${ }^{31}$ and treatment of comorbid pain may enhance outcomes of depression therapy. ${ }^{32}$

It is of interest that chest pain in men and neck or shoulder pain in women were the somatic symptoms most strongly associated with current suicidality score. Foldes-Busque et al. ${ }^{33}$ reported that panic-like anxiety is common in patients who suffer from unexplained chest pain, which was associated with depression and current suicidal risk. They assumed that repeated panic-like anxiety and the absence of an explanation and treatment for these symptoms could engender hopelessness, an emotion associated with suicide attempts. Previous studies consistently reported that comorbid MDD and panic disorder are associated with higher suicidal risk and current depression severity. ${ }^{34,35}$ In contrast, neck and shoulder pain are musculoskeletal symptoms which are commonly found in the general population, and are related to nonspecific psychological distress. ${ }^{36}$

This study also revealed that the more severe the chest pain, the higher the association with current suicidality score in men, and for women, neck or shoulder pain were associated with current suicidality score, although there were no significant differences in the rates of these types of pain between male and female MDD patients. Current suicidality score was associated with more than five of the somatic symptoms in men but not in women. These results suggest that current suicidality score is more strongly associated with somatic symptoms in men than in women. Although women more frequently express somatic symptoms than men in MDD patients, ${ }^{4}$ these findings suggest that more serious attention should be paid to male MDD patients who complain of chest pain and MDD patients who have multiple somatic symptoms, to prevent suicide.

\section{Limitations of the study}

There are several limitations of this study that should be considered when interpreting the findings. First, this study was a cross-sectional survey in which information about suicidal behaviors and somatic symptoms was based on retro- 
spective reports. Therefore, recall bias may have affected the accuracy of the results, particularly the incidence of false negative results. ${ }^{37}$ Second, patients may have been reluctant to expose their personal experiences relating to suicide and mental problems. ${ }^{38,39}$ Third, this study did not investigate personality disorders such as borderline personality disorder. Hostility may be associated with personality disorders. ${ }^{40}$ Fourth, the findings of our study may not be generalizable beyond Korean or Asian patient populations. It is possible that non-Asian populations express depressive symptoms differently, with less emphasis on somatic symptoms and more emphasis on other symptoms of depression. Finally, our study was unable to address potential biological underpinnings of the findings. We just emphasized on the patient's report of somatic symptoms, not focusing on the biological causes or origins of them.

\section{Conclusions}

In conclusion, MDD patients of both genders with suicidal ideation showed significantly more frequent and severe somatic symptoms than those without suicidal ideation. Pain was the somatic symptom most strongly associated with current suicidal risk in both genders. There was a gender difference in the somatic symptoms associated with current suicidality score, with chest pain in men, and neck or shoulder pain in women being associated with current suicidality score. Chest pain showed a severity-related increase of current suicidality in male MDD patients, but no such severity-related increase was found with neck or shoulder pain in females. To prevent suicide in MDD patients, we suggest that clinicians pay attention to patients' somatic symptoms, especially chest pain in men.

\section{Acknowledgments}

This study was supported by Pfizer Pharmaceuticals Korea Ltd. and the Basic Science Research Program through the National Research Foundation of Korea (NRF) of the Ministry of Education, Science and Technology (No. 2011-0013064). This study was also supported by a grant of the Korean Health Technology R\&D Project, Ministry of Health \& Welfare, Republic of Korea (A120051 suicide prevention research center).

\section{REFERENCES}

1. Simon GE, VonKorff M, Piccinelli M, Fullerton C, Ormel J. An international study of the relation between somatic symptoms and depression. N Engl J Med 1999;341:1329-1335.

2. American Psychiatric Association. Diagnostic and Statistical Manual of Mental Disorders (DSM-V), 5th ed. Washington, DC: American Psychiatric Press; 2013.

3. Kapfhammer HP. Somatic symptoms in depression. Dialogues Clin Neurosci 2006;8:227-239.

4. Wenzel A, Steer RA, Beck AT. Are there any gender differences in frequency of self-reported somatic symptoms of depression? J Affect Disord 2005;89:177-181.

5. Silverstein B. Gender differences in the prevalence of somatic versus pure depression: a replication. Am J Psychiatry 2002;159:1051-1052.
6. Dumais A, Lesage AD, Alda M, Rouleau G, Dumont M, Chawky N, et al. Risk factors for suicide completion in major depression: a case-control study of impulsive and aggressive behaviors in men. Am J Psychiatry 2005;162:2116-2124.

7. Stevens D, Wilcox HC, Mackinnon DF, Mondimore FM, Schweizer B, Jancic D, et al. Posttraumatic stress disorder increases risk for suicide attempt in adults with recurrent major depression. Depress Anxiety 2013;30:940-946.

8. Lopez de Lara C, Brezo J, Rouleau G, Lesage A, Dumont M, Alda M, et al. Effect of tryptophan hydroxylase-2 gene variants on suicide risk in major depression. Biol Psychiatry 2007;62:72-80.

9. Lopez de Lara C, Dumais A, Rouleau G, Lesage A, Dumont M, Chawky $\mathrm{N}$, et al. STin2 variant and family history of suicide as significant predictors of suicide completion in major depression. Biol Psychiatry 2006;59:114-120.

10. Agargun MY, Besiroglu L, Cilli AS, Gulec M, Aydin A, Inci R, et al. Nightmares, suicide attempts, and melancholic features in patients with unipolar major depression. J Affect Disord 2007;98:267-270.

11. Jeon HJ, Peng D, Chua HC, Srisurapanont M, Fava M, Bae JN, et al. Melancholic features and hostility are associated with suicidality risk in Asian patients with major depressive disorder. J Affect Disord 2013;148: 368-374.

12. Kim YK, Myint AM. Clinical application of low serum cholesterol as an indicator for suicide risk in major depression. J Affect Disord 2004;81: 161-166.

13. Jeon HJ, Hong JP, Fava M, Mischoulon D, Nyer M, Inamori A, et al. Childhood parental death and lifetime suicide attempt of the oppositegender offspring in a nationwide community sample of Korea. Suicide Life Threat Behav 2013;43:598-610.

14. Bohman H, Jonsson U, Von Knorring AL, Von Knorring L, Paaren A, Olsson G. Somatic symptoms as a marker for severity in adolescent depression. Acta Paediatr 2010;99:1724-1730.

15. Hagnell O, Rorsman B. Suicide and endogenous depression with somatic symptoms in the Lundby study. Neuropsychobiology 1978;4:180-187.

16. American Psychiatric Association. Diagnostic and Statistical Manual of Mental Disorders, 4th ed. Washington, DC: American Psychiatric Press; 1994.

17. Hamilton M. Development of a rating scale for primary depressive illness. Br J Soc Clin Psychol 1967;6:278-296.

18. Wei HT, Chen MH, Huang PC, Bai YM. The association between online gaming, social phobia, and depression: an internet survey. BMC Psychiatry 2012;12:92.

19. Hung CI, Wang SJ, Liu CY. Validation of the Depression and Somatic Symptoms Scale by comparison with the Short Form 36 scale among psychiatric outpatients with major depressive disorder. Depress Anxiety 2009;26:583-591.

20. Kim KW, Hong JP, Park SJ, Choi JH, Choi HR. Reliability and validity of Korean version of Depression and Somatic Symptom Scale (DSSS). Anxiety Mood 2011;7:9-15.

21. Sheehan DV, Lecrubier Y, Sheehan KH, Amorim P, Janavs J, Weiller E, et al. The Mini-International Neuropsychiatric Interview (M.I.N.I.): the development and validation of a structured diagnostic psychiatric interview for DSM-IV and ICD-10. J Clin Psychiatry 1998;59(Suppl 20):22-33.

22. Zhu C, Ou L, Geng Q, Zhang M, Ye R, Chen J, et al. Association of somatic symptoms with depression and anxiety in clinical patients of general hospitals in Guangzhou, China. Gen Hosp Psychiatry 2012;34:113120.

23. Haug TT, Mykletun A, Dahl AA. The association between anxiety, depression, and somatic symptoms in a large population: the HUNT-II study. Psychosom Med 2004;66:845-851.

24. Silverstein B, Patel P. Poor response to antidepressant medication of patients with depression accompanied by somatic symptomatology in the STAR*D Study. Psychiatry Res 2011;187:121-124.

25. Ilgen MA, Zivin K, McCammon RJ, Valenstein M. Pain and suicidal thoughts, plans and attempts in the United States. Gen Hosp Psychia- 
try 2008;30:521-527.

26. Scott KM, Hwang I, Chiu WT, Kessler RC, Sampson NA, Angermeyer $\mathrm{M}$, et al. Chronic physical conditions and their association with first onset of suicidal behavior in the world mental health surveys. Psychosom Med 2010;72:712-729.

27. Walker J, Waters RA, Murray G, Swanson H, Hibberd CJ, Rush RW, et al. Better off dead: suicidal thoughts in cancer patients. J Clin Oncol 2008;26:4725-4730.

28. Pompili M, Di Cosimo D, Innamorati M, Lester D, Tatarelli R, Martelletti P. Psychiatric comorbidity in patients with chronic daily headache and migraine: a selective overview including personality traits and suicide risk. J Headache Pain 2009;10:283-290.

29. Calandre EP, Vilchez JS, Molina-Barea R, Tovar MI, Garcia-Leiva JM, Hidalgo J, et al. Suicide attempts and risk of suicide in patients with fibromyalgia: a survey in Spanish patients. Rheumatology (Oxford) 2011; 50:1889-1893.

30. Pompili M, Innamorati M, Serafini G, Gonda X, Campi S, Rapinesi C, et al. How does subjective experience of pain relate to psychopathology among psychiatric patients? Gen Hosp Psychiatry 2012;34:534-540.

31. Karp JF, Scott J, Houck P, Reynolds CF 3rd, Kupfer DJ, Frank E. Pain predicts longer time to remission during treatment of recurrent depression. J Clin Psychiatry 2005;66:591-597.

32. Bair MJ, Robinson RL, Eckert GJ, Stang PE, Croghan TW, Kroenke K. Impact of pain on depression treatment response in primary care. Psychosom Med 2004;66:17-22.
33. Foldes-Busque G, Marchand A, Chauny JM, Poitras J, Diodati J, Denis I, et al. Unexplained chest pain in the ED: could it be panic? Am J Emerg Med 2011;29:743-751.

34. Brown LA, Gaudiano BA, Miller IW. The impact of panic-agoraphobic comorbidity on suicidality in hospitalized patients with major depression. Depress Anxiety 2010;27:310-315.

35. Diaconu G, Turecki G. Panic disorder and suicidality: is comorbidity with depression the key? J Affect Disord 2007;104:203-209.

36. Dyrehag LE, Widerstrom-Noga EG, Carlsson SG, Kaberger K, Hedner $\mathrm{N}$, Mannheimer C, et al. Relations between self-rated musculoskeletal symptoms and signs and psychological distress in chronic neck and shoulder pain. Scand J Rehabil Med 1998;30:235-242.

37. Patten SB. Recall bias and major depression lifetime prevalence. Soc Psychiatry Psychiatr Epidemiol 2003;38:290-296.

38. de Graaf R, Bijl RV, Smit F, Ravelli A,Vollebergh WA. Psychiatric and sociodemographic predictors of attrition in a longitudinal study: The Netherlands Mental Health Survey and Incidence Study (NEMESIS). Am J Epidemiol 2000;152:1039-1047.

39. Eaton WW, Anthony JC, Tepper S, Dryman A. Psychopathology and attrition in the epidemiologic catchment area surveys. Am J Epidemiol 1992;135:1051-1059.

40. Wilson ST, Stanley B, Oquendo MA, Goldberg P, Zalsman G, Mann JJ.Comparing impulsiveness, hostility, and depression in borderline personality disorder and bipolar II disorder. J Clin Psychiatry 2007;68:15331539. 